\title{
Predicted Dark Matter Quantitation in Flat Space Cosmology
}

\author{
Eugene Terry Tatum \\ 760 Campbell Ln. Ste. 106 \#161, Bowling Green, KY 42104, USA \\ Email:ett@twc.com
}

How to cite this paper: Tatum, E.T. (2018) Predicted Dark Matter Quantitation in Flat Space Cosmology. Journal of Modern Physics, 9, 1559-1563.

https://doi.org/10.4236/jmp.2018.98096

Received: June 25, 2018

Accepted: July 15, 2018

Published: July 18, 2018

Copyright $\odot 2018$ by author and Scientific Research Publishing Inc. This work is licensed under the Creative Commons Attribution International License (CC BY 4.0).

http://creativecommons.org/licenses/by/4.0/

\section{(c) (i) Open Access}

\begin{abstract}
The purpose of this paper is to show how the dark matter predictions of FSC differ with respect to the standard cosmology assertion of a universal dark matter-to-visible matter ratio of approximately 5.3-to-1. FSC predicts the correct ratio to be approximately 9-to-1, based primarily on the universal observations of global spatial flatness in the context of general relativity. The FSC Friedmann equations incorporating a Lambda $\Lambda$ cosmological term clearly indicate that a spatially flat universe must have equality of the positive curvature (matter mass-energy) and negative curvature (dark energy) density components. Thus, FSC predicts that observations of the Milky Way and the nearly co-moving galaxies within 100 million light years will prove the 5.3to-1 ratio to be incorrect. The most recent galactic and perigalactic observations indicate a range of dark matter-to-visible matter ratios varying from essentially zero (NGC 1052-DF2) to approximately 23-to-1 (Milky Way). The latter ratio is simply astonishing and promises an exciting next few years for astrophysicists and cosmologists. Within the next few years, the mining of huge data bases (especially the Gaia catalogue and Hubble data) will resolve whether standard cosmology will need to change its current claims for the cosmic energy density partition to be more in line with FSC, or whether FSC is falsified. A prediction is that standard cosmology must eventually realize the necessity of resolving the tension between their flatness observations and their assertion of dark energy dominance. The author makes the further prediction that FSC will soon become the new paradigm in cosmology.
\end{abstract}

\section{Keywords}

Flat Space Cosmology, Standard Cosmology, Cosmology Theory, Dark Matter, Cosmic Microwave Background, Planck Collaboration, Gravitational Entropy, Black Holes 


\section{Introduction and Background}

In sharp contrast to standard cosmology, Flat Space Cosmology (FSC) makes quite a number of predictions which would invalidate the theory if proven false. Many of these predictions can be derived from the FSC Friedmann equation which must always hold true in FSC [1]:

$$
\frac{3 H^{2} c^{2}}{8 \pi G} \cong \frac{\Lambda c^{4}}{8 \pi G}
$$

Wherein the left-hand term is the total matter energy density and the right-hand term is the dark energy density. The $H^{2}$ symbol is the squared Hubble parameter value in metric units $\left(s^{-2}\right)$ and the $\Lambda$ symbol is the cosmological parameter in metric units $\left(\mathrm{m}^{-2}\right)$. In a globally spatially flat expanding universe, which we observe, general relativity stipulates that the global positive curvature of total matter mass-energy density must equate with the global negative curvature of dark energy density. If the case were otherwise, the universe would have a global spatial curvature of sign and magnitude corresponding to the dominating energy density, which we do not observe. Thus, when the universe is at Friedmann's critical density, as appears to be the case by astronomical observations [2], FSC stipulates that $50 \%$ of the critical density must be attributable to total matter (visible matter plus dark matter) and $50 \%$ of the critical density must be attributable to dark energy.

One of the longstanding observational facts is that the visible matter of our universe comprises only about $5 \%$ of the critical density. Thus, FSC predicts a dark matter-to-visible matter ratio of approximately $45 / 5$ or 9-to- 1 . As detailed in the Planck Collaboration consensus report, the ratio of dark matter-to-visible matter is claimed to be approximately 5.3-to-1. However, so little is currently known about precisely detecting and quantifying dark matter that this ratio is subject to higher revision in the likely event that more dark matter is discovered in the future. For this reason, the Planck Collaboration ratio must be considered as a constraint only on the low end.

Galactic and perigalactic distributions of dark matter can be surprisingly variable, as evidenced by the 29 March 2018 report in Nature [3] of an exceedingly diffuse distant galaxy (NGC 1052-DF2) apparently completely lacking in dark matter! Hence, the global (i.e., CMB) Planck Collaboration ratio of 5.3-to-1 cannot dogmatically be considered even an approximation of all galactic and perigalactic ratios, particularly if these ratios are scalar over cosmic time.

What is now required is a best estimate of the co-moving dark matter-tovisible matter ratio within approximately 50 - 100 nearby galaxies. The only observable truly co-moving galaxy for us is the Milky Way galaxy itself. All other galaxies are observationally displaced in distance and time to some degree. However, all galaxies within 100 million light years of the Milky Way should be sufficiently close to us to be considered approximate co-movers for the required observations. These are the galaxies of the Virgo Supercluster. There are 160 galaxy groups within 100 million light years of the Milky Way galaxy. The number 
of large galaxies is approximately 2500 and the number of dwarf galaxies is approximately 25,000 [4]. Data mining of the Gaia catalogue will not only allow researchers to determine the range of dark matter-to-visible matter ratios within the most accessible nearby co-moving galaxies, but to determine the average dark matter-to-visible matter ratio. The average ratio may be calculated by dividing the sum of the dark matter numerators by the total number of nearby co-moving galaxies reliably measured. As mentioned, FSC predicts this average ratio to be very close to 9-to-1. A radically different average co-moving ratio would falsify FSC. Standard cosmology, on the other hand, has no capacity to predict this ratio. Therefore, whatever this average co-mover ratio turns out to be, it will be inserted into the standard inflationary model after its determination.

Given the recent report of the galaxy apparently devoid of dark matter, astronomers around the world are scrambling to mine the Gaia catalogue data for further clues with respect to dark matter. The most logical place to start, of course, is with the Milky Way galaxy. Remarkably, this data has just become available! This author predicts that Posti and Helmi's May 2018 arXiv.org publication of "Mass and Shape of the Milky Way's Dark Matter Halo with Globular Clusters from Gaia and Hubble" [5] will be considered a landmark publication concerning galactic and perigalactic dark matter. This study reveals that the virial volume comprising our Milky Way Galaxy and its dark matter halo has a dark matter-to-visible matter ratio of approximately 23.074-to-1. Thus, the matter confined within the halo radius ("virial radius") of our Milky Way galaxy appears to be approximately $95.85 \%$ dark matter and $4.15 \%$ visible matter!

So that the reader can make the same calculations, the relevant measurements made by Posti and Helmi are repeated here: the virial mass is reported to be 1.3 $+/-0.3 \times 10^{12}$ solar masses; the mass of the Milky Way galaxy within a generous $20 \mathrm{kpc}$. (a radius of approximately 65,200 light years) is reported to be $1.91 \times$ $10^{11}$ solar masses, of which Posti and Helmi attribute $1.37 \times 10^{11}$ solar masses to intragalactic dark matter. One can, therefore, assume the remaining $0.54 \times 10^{11}$ solar masses to be the galactic visible matter within approximately 65,200 light years of the Milky Way center, which can be safely assumed to be greater than $99 \%$ of the Milky Way visible matter. This is because numerous reliable sources indicate the visible matter of the Milky Way to be within a radius of 50,000 light years of the galactic center. Posti and Helmi measure a virial radius of $287 \mathrm{kpc}$. This is greater than 14 times their defined radius of the galactic disc, and surely must encapsulate the vast majority of the Milky Way dark matter. Hence, one can assume that the ratio of $1.3 \times 10^{12}$ solar masses to $0.54 \times 10^{11}$ solar masses is an excellent approximation of the Milky Way dark matter-to-visible matter ratio. This is how the author calculated the ratio and percentage numbers in the prior paragraph.

These very recent observations ranging from $0 \%$ galactic and perigalactic dark matter (NGC 1052-DF2) to $95.85 \%$ dark matter within the virial volume of the Milky Way galaxy must be somewhat jarring to standard model proponents. The 
current dogmatic acceptance of approximately 30\% universal total matter mass-energy and approximately $70 \%$ dark energy appears to be on a shaky foundation [6]-[11]. Within the next few years, the mining of huge data bases (especially the Gaia catalogue and Hubble data) will resolve whether standard cosmology will need to change its current claims for the cosmic energy density partition to be more in line with FSC, or whether FSC is falsified. Regardless, standard cosmology must eventually realize the necessity of resolving the tension between their flatness observations and their current assertion of dark energy dominance.

\section{Summary and Conclusions}

The purpose of this paper has been to show how the dark matter predictions of FSC differ with respect to the standard cosmology assertion of a universal dark matter-to-visible matter ratio of approximately 5.3-to-1. FSC predicts the correct ratio to be approximately 9-to-1, based primarily on the universal observations of global spatial flatness in the context of general relativity. The FSC Friedmann equations incorporating a Lambda $\Lambda$ cosmological term clearly indicate that a spatially flat universe must have equality of the global positive curvature (matter mass-energy) and global negative curvature (dark energy) density components. Thus, FSC predicts that observations of the Milky Way and the nearly co-moving galaxies within 100 million light years will prove the 5.3-to-1 ratio to be incorrect. The most recentgalactic and perigalactic observations indicate a range of dark matter-to-visible matter ratios varying from essentially zero (NGC 1052-DF2) to approximately 23-to-1 (Milky Way). The latter ratio is simply astonishing and promises an exciting next few years for astrophysicists and cosmologists. Within the next few years, the mining of huge data bases (especially the Gaia catalogue and Hubble data) will resolve whether standard cosmology will need to change its current claims for the cosmic energy density partition to be more in line with FSC, or whether FSC is falsified. A prediction is that standard cosmology must eventually realize the necessity of resolving the tension between their flatness observations and their assertion of dark energy dominance. The author makes the further prediction that FSC will soon become the new paradigm in cosmology.

\section{Dedications and Acknowledgements}

This paper is dedicated to Dr. Stephen Hawking and Dr. Roger Penrose for their groundbreaking work on black holes and their possible application to cosmology. Dr. Tatum also thanks Dr. Rudolph Schild of the Harvard Center for Astrophysics for his past support and encouragement.

\section{References}

[1] Tatum, E.T. and Seshavatharam, U.V.S. (2018) Journal of Modern Physics, 9, 1404-1414. https://doi.org/10.4236/jmp.2018.97085 
[2] Planck Collaboration XIII. (2016) Astronomy \& Astrophysics, 594, A13. http://arxiv.org/abs/1502.01589

[3] van Dokkum, P., et al. (2018) Nature, 555, 629-632. https://doi.org/10.1038/nature25767

[4] Theuns, T. (2003) The Universe within 100 Million Light Years. Institute of Computational Cosmology. http://www.icc.dur.ac.uk/ tt/Lectures/Galaxies/LocalGroup/Back/virgo.html

[5] Posti, L. and Helmi, A. (2018) Mass and Shape of the Milky Way's Dark Matter Halo with Globular Clusters from Gaia and Hubble. arXiv:1805.01408v1 [astro-ph.GA]

[6] Tatum, E.T. and Seshavatharam, U.V.S. (2018) Journal of Modern Physics, 9, 1397-1403. https://doi.org/10.4236/jmp.2018.97084

[7] Tutusaus, I., et al. (2017) Astronomy \& Astrophysics, 602, A73. arXiv:1706.05036v1 [astro-ph.CO].

[8] Dam, L.H., et al. (2017) Apparent Cosmic Acceleration from Type Ia Supernovae. Monthly Notices of the Royal Astronomical Society. arXiv:1706.07236v2 [astro-ph.CO].

[9] Nielsen, J.T., et al. (2015) Scientific Reports, 6, Article number 35596. arXiv:1506.01354 [astro-ph.CO].

[10] Wei, J.-J., et al. (2015) Astronomical Journal, 149, 102-113. https://doi.org/10.1088/0004-6256/149/3/102

[11] Melia, F. (2012) Astronomical Journal, 144. arXiv:1206.6289 [astro-ph.CO] https://doi.org/10.1088/0004-6256/144/4/110 